\title{
The association of knowledge on oral health and utilization of dental services among pregnant women
}

\author{
Ola Mousa*1, Asmaa Hamed ${ }^{1}$, Norah Al Omar ${ }^{2}$ \\ ${ }^{1}$ College of Nursing, Minia University, Minia, Egypt \\ ${ }^{2}$ College of Applied Medical Sciences, King Faisal University, KSA
}

Received: August 17, 2018

Accepted: January 14, 2019

Online Published: January 29, 2019

DOI: $10.5430 /$ jnep.v9n6p1

URL: https://doi.org/10.5430/jnep.v9n6p1

\begin{abstract}
Good oral health during pregnancy can possibly reduce complications and this will improve the quality of life of the pregnant woman. In addition, this will prevent the risk of the child to develop early childhood dental caries in the future. The main objective of this study was to determine if there is an association existingbetween the level of knowledge on oral health and utilization of dental services among pregnant women. Furthermore, the study determined the (a) proportion among pregnant women who had high level of knowledge regarding dental careduring pregnancy, (b) proportion of pregnant women who ever visited the dentist during pregnancy, (c) proportions of expecting women who are regularly brushing her teeth and whouses dental floss during pregnancy, and (d) factors that hinders the expecting women from visiting the dentist during pregnancy. A descriptive cross-sectional study was conducted at El Minia University Maternal and Child Health Hospital, El Minia, Egypt. A cross-sectional self-administered questionnaire-based survey was conducted among 200 expectant mothers, approached 156 out of them returned completed questionnaires, representing a response rate of $78 \%$. Results revealed that: (a) nearly $65 \%$ of pregnant mothers had a high level of poor knowledge regarding dental care during pregnancy, (b) roughly $24 \%$ of pregnant women ever visited the dentist during pregnancy, (c) about $80 \%$ and $43 \%$ of expecting women regularly brush their teeth at least twice per day and use dental floss during pregnancy, respectively, and (d) among the factors that hinder the expecting women from visiting the dentist during pregnancy include having no time to visit, the consultation cost, fear and anxiety about the procedure, long waiting time, transportation problem and the idea of not needing the dental care. Moreover, the results showed that there was no sufficient evidence to say that the level of knowledge regarding dental service during pregnancy is associated with the utilization of dental service during pregnancy (Pearson Chi-square $p$-value $=.160$ ). The study concludes that health promotion on oral care during prenatal visits should address the mythologies and misconceptions among pregnant women. Therefore, healthcare providers should raise awareness among expectant mothers about giving special attention to oral health needs due to the higher risk of oral diseases that may affect pregnancy and the child inside the womb.
\end{abstract}

Key Words: Pregnancy, Oral hygiene, Knowledge, Practices, Barriers

\section{INTRODUCTION}

The importance of oral health during pregnancy has hearkened the awareness of different policy makers, various foundations/agencies and health care providers who serve pregnant women and young children in the past decade.They have recognized that oral health is an integral part of preventive health care for pregnant women and their newborns. ${ }^{[1]}$

A pregnant woman is particularly associated with numerous physiological and emotional changes. During this time, many

*Correspondence: Ola Mousa; Email: olaessam1977@yahoo.com; Address: College of Nursing, Minia University, Minia, Egypt. 
changes happen within the oral cavity that can be connected to periodontal illness, including gingivitis and periodontitis. Studies have demonstrated that there is an association between increased plasma levels of pregnancy hormones and decay in periodontal wellbeing status. ${ }^{[2]}$ Nearly $60 \%$ to $75 \%$ of pregnant women have gingivitis. ${ }^{[3]}$ Various pathologies concerning oral cavity were conveyedby expectant mothers. $^{[4]}$

Maintaining exemplary oral hygiene during pregnancy is vital in reducing any untoward signs and symptoms of both mother and infant. It can possibly reduce complications and improve the quality of life of pregnant women. In addition, this will prevent the risk of the child to develop early childhood dental caries in the future. ${ }^{[5]}$ Therefore, it is during this time that women should be a keen observer for any health changes to maintain an optimum level of wellness. ${ }^{[6]}$

With the importance of oral health for this population, expectant mothers should be the priority population group for dental care, considering several factors of this period (1) they may acquire oral alterations during the gestational stage; (2) they may have accrued needs that might put at risk the health of both the mother and child; (3) it is essential for the pregnant mothers to include in the health education programs since they influence the family's eating and hygiene habits as they establish mindsets within the household system; (4) pregnant women can be involved in the scheduled periodic programs since they are regularly available in the healthcare facilities during this period, thus, they compromise an easy access group. ${ }^{[7]}$

Pregnant women and the members of the health care team like a dentist should increase their knowledge and make safety elucidation on a safe dental treatment regimen.Dental care during pregnancy is always safe when proper guidelines on treatment modalities are followed appropriately. ${ }^{[8]}$ In relation to this, the 2013 Committee Conclude from the American College of Obstetricians and Gynecologists suggests that all health care providers evaluate oral wellbeing at the primary ante-birth visit. ${ }^{[1]}$ Also, the Health Resources and Services Administration(HRSA) (2014) integrated oral health and primary care practice. ${ }^{[9]}$ Itsummarized skilled oral health core clinical capabilities suitable for primary care providers, containing but not restricted to NPs, NMs, MDs, DOs, and Pas. ${ }^{[9]}$

It is also essential to recognize the pregnant women's oral health awareness and behaviors to distinguish barriers to accessing care and to have suggestions for the improvement of oral health information. This will aid in the role of an expectant mother in the oral health of her children and the importance of good oral hygiene during pregnancy. ${ }^{[10]}$
Moreover, mothers need to have positive attitudes and greater knowledge regarding oral habits to promote their child's oral health condition. Mothers with a consistent source of dental care are expected to take their offspring to the dentist and to develop approaches that promote good oral health for themselves and their children. ${ }^{[11]}$ Hence, they play a vital role in the preservation of their children's oral hygiene. ${ }^{[12]}$

However, while dental treatment is an important part of the avoidance and treatment of oral diseases, getting and using dental services are not common or known among the public. ${ }^{[13-15]}$

With this, the main objective of the study was to determine if there is an association existingbetween the level of knowledge on oral health and utilization of dental services among pregnant women. Furthermore, the study determined the (a) proportion of pregnant women who had high level of knowledge regarding dental care during pregnancy, (b) proportion of pregnant women who visited the dentist during pregnancy, (c) proportions of expecting women who are regularly brushing her teeth and who uses dental floss during pregnancy, and (d) factors that hinders the expecting women from visiting the dentist during pregnancy.

\section{MATERIALS AND METHODS}

\subsection{Study design}

The study is descriptive cross-sectional, conducted in El Minia University Maternal and Child Health Hospital, El Minia, Egypt. It was conducted upon the approval from the Head of El Minia University Maternal and Child Health Hospital, and from the ethical committee of the Department of Obstetrics and Gynecology, Minia University Hospital, on April 10, 2016.

The study participants were randomly selected based on convenience method from those who were visiting the Outpatient Clinic of El Minia University Maternal and Child Health Hospital during a period of 2 months, August 2 to October 2,2016 . Informed consent was obtained from each of the participants prior to conducting the study and asking them to complete a self-administered questionnaireaddressing the various aspects of expectant mothers' knowledge, beliefs and attitude about oral health and their own oral hygiene practices. The sample inclusion criteria were: all pregnant women, regardless of age, educational, and social class. Absenteeism, unwillingness to participate, non-pregnant, or participants younger than 18 years old were excluded from the criteria. Among 200 expectant mothers in El Minia University Maternal and Child Health Hospital who were given with self-administered questionnaire-based survey, 156 of them returned the completed questionnaires, representing a 
response rate of $78 \%$.

The questionnaire-based surveywas prepared by the researchers in English and in Arabic. The questions were developed after referring to relevant literature to assess the participants' knowledge, beliefs and health practices regarding oral hygiene for themselves and their children. The questionnaireswere tested for validity and reliability with 20 women and modified the questionnaire based on the findings. The final questionnaires are available in English and Arabic and ready to use by study staff members. It was read to women who were illiterate.

The questionnaire has four sections. The first part consists of the socio-demographic profile of the respondents such as their age, educational attainment, the gestational age and the number of pregnancies. The next part includes eleven (11) questions related to knowledge of oral health. According to the mean score, the knowledge score was regrouped into two (2) categories as follows: 1) high level of knowledge, 2) low level of knowledge. Therefore, a score of seven (7) and above was rated as good knowledge, while six (6) and below falls under poor knowledge. The third section contained eight (8) issues related to dental health beliefs, which was set to survey the respondents' attitudes toward oral health. The expectant mothers were requested to indicate their preference as to whether they "strongly agree, agree, neutral or undecided, disagree and strongly disagree" in every statement. Their attitude towards oral health will be computed for respondents by determining the total number of acquired positive oral health attitude. The maximum achievable score was eight (8) which means that a higher score indicates an extra positive attitude. Respondents who have a score of six (6) and above were graded as having a positive attitude towards oral health. In addition, the fourth (4th) section covered the assessment of the respondent's daily oral health practices and dental health services utilization during pregnancy. The total score was calculated for each participant and the subjects were divided based on the number of correct answers into a low level of knowledge (score $<12$ ), and high level of knowledge (score 13 and above).

For the statistical analysis, proportions among pregnant women who had a high level of knowledge regarding dental care during pregnancy, who visited the dentist during pregnancy, who regularly brushtheir teeth, and who uses dental floss during pregnancy were calculated using STATA MP version 14. The frequencies of the factors that hinder the expecting women from visiting the dentist during pregnancy were also computed. Pearson Chi-square test was used to determine if an association exists between the level of knowledge on oral health and utilization of dental services among pregnant women.

\section{RESULTS}

\subsection{Socio-demographic characteristics}

Out of 156 respondents, about $53.21 \%$ were between the ages of 21 and 30 years. The median age was 24 years with an interquartile range of 9 years. The youngest respondent was 19 years old and the oldest was 45 years old. The distributionsof respondents according to age are shown in Figures $1 \& 2$.

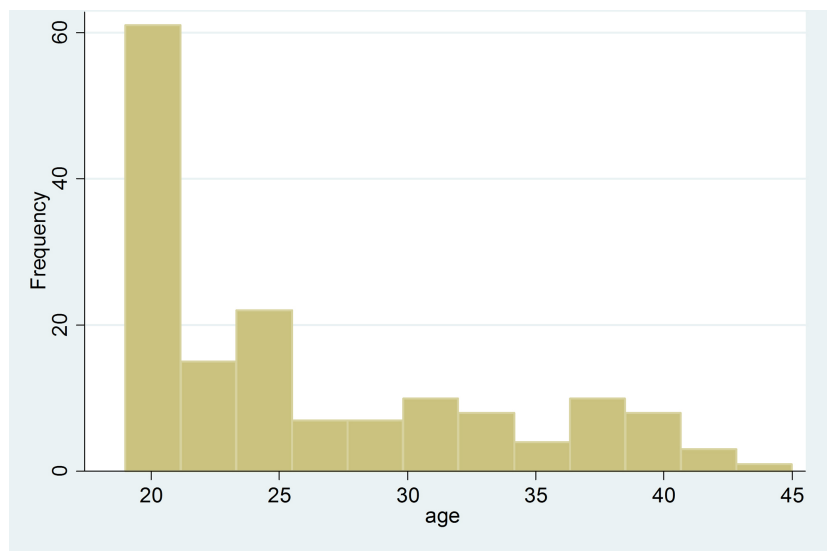

Figure 1. Distribution of respondents according to age

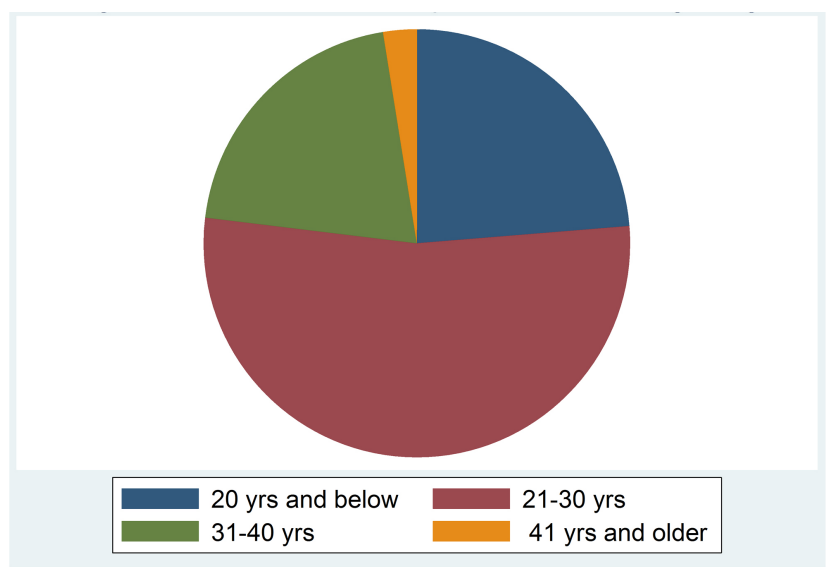

Figure 2. Distribution of respondents according to age

About $60 \%$ of the respondents were high school graduates, $85 \%$ were in the middle-income class, $52 \%$ were in their third trimester of pregnancy, and $58 \%$ were privately paying for the expenses during pregnancy. Roughly $31 \%$ were first-time mothers.

Table 1 shows the distribution of respondents according to educational attainment, economic status, number of children, trimestersof pregnancy and source of payment. 
Table 1. Distribution of respondents according to educational attainment, socio-economic status, number of children, trimester of pregnancy, and source of payment

\begin{tabular}{lll}
\hline Variable & Count (n) & Percentage (\%) \\
\hline Educational Attainment & & \\
College graduate & 44 & 28.2 \\
High school graduate & 94 & 60.3 \\
Elementary school graduate & 14 & 9 \\
None & 4 & 2.6 \\
Economic status & & \\
Low-income & 2 & 1.2 \\
Middle-income & 133 & 85.3 \\
High -income & 21 & 13.5 \\
Number of children & & \\
0 & 49 & 31.4 \\
1 & 39 & 25 \\
2 & 25 & 16 \\
3 & 22 & 14.1 \\
4 and more & 21 & 13.5 \\
Pregnancy Trimester & & \\
1st & 35 & 22.4 \\
2nd & 40 & 25.6 \\
3rd & 81 & 52 \\
Source of payment & & \\
Public/Governmental & 66 & 42.3 \\
Private & 90 & 57.7 \\
\hline
\end{tabular}

\subsection{Proportion of expecting women according to oral health knowledge and practices}

There were $65 \%$ or 6 in every 10 expecting women who possess a high level of knowledge regarding dental care during pregnancy while about $24 \%$ or 1 in every 4 expecting women visits or visited the dentist during pregnancy. Eight in every ten or $80 \%$ of the expecting women are brushing their teeth at least twice every day and 4 in every 10 expecting women or $43 \%$ are using dental floss during pregnancy.

\subsection{Factors that hinder the expecting women from visit- ing the dentist during pregnancy}

About 37/156 of the respondents said that they do not have the time to visit the dentist during pregnancy because of being busy with other things. For 32/156 respondents, the cost of the dental service discourages them to avail it. Fear and anxiety about the dental procedure, long waiting time and the idea of not needing dental care during pregnancy are the reasons given by 21 of the 156 respondents. About 8 respondents claimed that because of transportation problems they were not able to visit the dentist.

\subsection{Association between the level of knowledge regard- ing dental service during pregnancy and the utiliza- tion of dental service during pregnancy}

It is specified that association between the level of knowledge regarding dental service during pregnancy and the utilization of dental service during pregnancy is not sufficient as supported by Pearson Chi-square $p$-value $=.160$.

Table 2. Proportion of expecting women according to oral health knowledge and practices

\begin{tabular}{lll}
\hline Proportion of expecting women & Count (n = 156) & Percentage (\%) \\
\hline Who possess high level of knowledge on dental care during pregnancy? & 101 & 65 \\
Who ever visited the dentist during pregnancy? & 37 & 24 \\
Who are regularly brushing her teeth? & 125 & 80 \\
Who use dental floss during pregnancy? & 67 & 43 \\
\hline
\end{tabular}

Table 3. Factors that hinder the expecting women from visiting the dentist during pregnancy

\begin{tabular}{lll}
\hline Factors & Count (n = 156) & Percentage (\%) \\
\hline Lack of time & 37 & 24 \\
Cost of Dental services & 32 & 21 \\
$\begin{array}{l}\text { Fear and anxiety about the dental procedure, long waiting time and the idea of not } \\
\text { needing dental care during pregnancy }\end{array}$ & 21 & 13 \\
Transportation problem & 8 & 5 \\
\hline
\end{tabular}

\section{Discussion}

In an emerging nation like Egypt, it is necessary to design an efficient antenatal dental health education program to maintain a broad knowledge of the maternal oral hygiene practices and attitude about pregnancy on oral health.

The study found out that $65 \%$ of the women had good knowledge and attitude toward oral health. Women believe that it 
is essential to have healthy teeth knowing the relationship of sweet foods and dental cavities had some impact on oral health. This result was similar to Boggess et al. (2010) who discussed that pregnant women had some knowledge about oral health contrary to the findings of Bamanikar (2013) who stated that the knowledge related to dental care was poor among pregnant women. ${ }^{[16,17]}$

Among the participants, 57\% are awareof the need to perform regular dental visits to prevent dental health problems, however, only $24 \%$ of expectant mothers had consulted a dentist during pregnancy on a regular basis. This means that awareness is not always translated to practice.These findings are similar to Bamanikar (2013) wherein a majority of the pregnant women (96.8\%) agreed that women should have a dental checkup during pregnancy, but only $55.9 \%$ had done it for the current pregnancy. ${ }^{[17]}$ Furthermore, this is also supported by the study in India conducted by Ganesh et al. (2011), which mentioned that only $4 \%$ of the women had regular dental visits. ${ }^{[18]}$ Also, Boggess et al. (2010) found out that participants' oral hygiene practices were associated with routine dental care utilization during pregnancy and the prevalence of informed dental visits during pregnancy amongst their study population was 25 percent. ${ }^{[16]}$ Lack of information or proper access to health care facilities that disseminate appropriate oral health and hygiene during pregnancy can be the probable reason. This result brings out the significance of educating the expectant mothers since they look after their own health and their infants. Studies conducted by Shenoy and Chacko stated that pregnant women in Karnataka, India has reportedly having a poor knowledge and practices in oral health hygiene. ${ }^{[19]}$

Other studies have reported the rate of attendance in dental clinics as $50 \%$, mainly due to work commitment. However, $87.8 \%$ of them had a positive attitude towards having a checkup soon. This is in contrast to a similar study done by Hajikazemi (2008) in Iran, were 70\% ( $n=224)$ of pregnant women had a negative attitude of having a dental check-up in the future. ${ }^{[20]}$

The result of the study revealedthat lack of time and money, their belief that it is not necessary to have an oral health, their fear and anxiety about the dental procedure, long waiting time are the primary barriers for pregnant women in consulting a dentist during pregnancy. According to Marchi K (2010), among the reasons why pregnant women don't go to dentistry are economic problems, not having a concern about the teeth, not giving importance on its the effects on pregnancy, and not gettingthe service. ${ }^{[21]}$ Moreover, Bamanikar (2013), mentioned that the common supposed barriers were long waiting time at the government clinics $(53.7 \%)$, space from home to the clinics (24.4\%) and negative approaches of medical workers (9.8\%). ${ }^{[17]}$ Another study in Australiareported that among mothers reporting a dental problem, prenatal care, or PNC, insurance through public funding and late PNC entry were significantly associated with their not getting dental care. ${ }^{[14]}$

The present study also found out that there is no association between the level of knowledge regarding dental service and the utilization of dental service during pregnancy. Though many health professionals are aware of the importance of oral health, often they do not address it as part of their provision of preconception, prenatal, or well-woman care. ${ }^{[22,23]}$ Hashim and Akbar found that $95.4 \%$ of gynecologists surveyed had knowledge about the association between oral health and pregnancy and that $85.2 \%$ recommended dental visits for their patients, however, in Cigna Corporation (2015) survey, theyreported that only $44 \%$ of women said that their doctor talked to them about oral health during their pregnancy visits. $^{[22,24]}$

\section{Conclusion}

There is no substantial evidence to show the association between the association of knowledge on oral health and utilization of dental services among pregnant women. Though the majority of the participants have a high level of knowledge on dental care, there is a minimal number who visited the dentist during pregnancy. This is due to some factors including lack of time, cost of dental services and fear and anxiety about the dental procedure, long waiting time and the idea of not needing dental care during pregnancy. Due to the higher risk of oral diseases that may affect pregnancy and the child inside the womb, healthcare providers should raise awareness among expectant mothers about giving special attention to oral health needs and its importance. Health promotion on oral care during prenatal visits should address the mythologies and misconceptions among pregnant women.Oral health promotion to women should use available and appropriate ways, creative, consistent and comprehensive communication strategies.

\section{CONFlicts of InTEREST Disclosure}

The authors declare that there is no conflict of interest statement. 


\section{REFERENCES}

[1] American College of Obstetricians and Gynecologists Women's Health Care Physicians Committee on Health Care for Underserved Women. Committee opinion no. 569: Oral health care during pregnancy and through the lifespan. Obstetrics and Gynecology. 2013; 122(2 Pt. 1): 417-422. PMid:23969828

[2] Wu M, Chen SW, Jiang SY. Relationship between gingival inflammation and pregnancy. Mediators of Inflammation. 2015.

[3] American Dental Association Council on Access, Prevention, and Interprofessional Relations. Women's oral health issues [white paper] 2006.

[4] Annan BDRT, Nuamah K. Oral pathologies seen in pregnant and nonpregnant women. Ghana Med. J. 2005; 39(1): 24-27. PMid:17299537

[5] Gambhir RS, Gupta T, Sekhon TS, et al. Oral health knowledge and awareness among pregnant women in India: A systematic review. J Indian socperiodontol. 2015 Nov-Dec; 19(6): 612-617.

[6] Silk H, Douglass AB, Douglass JM, et al. Oral health during pregnancy. Am Fam Physician. 2008; Apr; 77(8): 1139-1144.

[7] Leal NP. Oral health of pregnant women: knowledge, practices and representations of the doctor, dentist and patient. Rio de Janeiro: Oswaldo Cruz Foundation; 2006.

[8] Oral Health Care During Pregnancy Expert Workgroup. Oral health care during pregnancy: A national consensus statement-Summary of an expert workgroup meeting. 2012. Available from: http://mchoralhealth.org/PDFs/OralHealthP regnancyConsensus.pdf

[9] U.S. Department of Health and Human Services, Health Resources and Services Administration. Integration of oral health and primary care practice. 2014. Available from: http://www.hrsa.gov/pub lichealth/clinical/oralhealth/primarycare/

[10] Rigo L, Dalazen J, and Garbin RR. Impact of dental orientation given to mothers during pregnancy on oral health of their children. Einstein (Sao Paulo). 2016 Apr-Jun; 14(2): 219-225. PMid:27462897 https ://doi.org/10.1590/S1679-45082016A03616

[11] Grembowski D, Spiekerman C, Milgrom P. Disparities in a regular source of dental care among mothers of Medicaid-enrolled preschool children. J Health Care Poor Underserved. 2007; 18(4): 789-813. PMid:17982208 https ://doi.org/10.1353/hpu.2007.0096

[12] Manchanda K, Sampath N, Sarkar A. Evaluating the effectiveness of oral health education program among mothers with 6-18 months children in prevention of early childhood caries. Contempclin Dent. 2014; 5(4): 478-483. PMid:25395763 https ://doi .org/10.410 3/0976-237X. 142815
[13] Hughes E, McCracken M, Roberts H, et al. Surveillance for certain health behaviors among states and selected local areas: behavioral risk factor surveillance system, United States, 2004. MMWR SurveillSumm. 2006; 55(7): 1-124.

[14] Gaffield ML, Gilbert BJ, Malvitz DM, et al. Oral health during pregnancy: an analysis of information collected by the pregnancy risk assessment monitoring system. JADA. 2001; 132(7): 1009-1016.

[15] Ressler-Maerlender J, Krishna R, Robison V. Oral health during pregnancy: current research. J Womens Health (Larchmt). 2005; 14(10): 880-882. PMid:16372888 https://doi.org/10.1089/ jwh. 2005.14.880

[16] Boggess KA, Urlaub DM, Massey KE, et al. Oral hygiene practices and dental service utilization among pregnant women. J Am Dent Assoc. 2010; 41(5): 553-561. https://doi.org/10.14219/jad a.archive. 2010.0228

[17] Bamanikar S, Kee L. Knowledge, Attitude and Practice of Oral and Dental Healthcare in Pregnant Women. Oman Med J. 2013 Jul; 28(4): 288-291. PMid:23904926 https : //doi.org/10.5001/om j. 2013.80

[18] Ganesh A, Ingle NA, Chaly PE, et al. A survey on dental knowledge and gingival health of pregnant women attending government maternity hospital, Chennai. J Oral Health Comm Dent. 2011; 5: 24-30.

[19] Chacko V, Shenoy R, Prasy HE, et al. Self-reported awareness of oral health and infant oral health among pregnant women in Mangalore, India-A prenatal survey. Int J Health Rehabil Sci. 2013; 2: 109-15.

[20] Hajikazemi E, et al. The relationship between Knowledge, Attitude and Practice of Pregnant Women about Oral and Dental Care. European Journal of Scientific Research. 2008; 24(4): 556-562.

[21] Marchi K, Owons S, Braveman B, et al. Most Pregnant Women in California Do Not Receive Dental Care: Findings from a PopulationBased Study. Public Health Rep. 2010 Nov-Dec; 125(6): 831-842.

[22] Hashim R, Akbar M. Gynecologists' knowledge and attitudes regarding oral health and PD leading to adverse pregnancy outcomes. Journal of International Society of Preventive \& Community Dentistry. 2014; 4(Suppl. 3): S166-S172.

[23] Morgan MA, Crall J, Goldenberg RL, et al. Oral health during pregnancy. The Journal of Maternal-Fetal \& Neonatal Medicine. 2009; 22(9): 733-739. PMid:19488943 https ://doi.org/10.3109/14 767050902926954

[24] Cigna Corporation. Healthy smiles for mom and baby: Insights into expecting and new mothers' oral health habits. 2015. 